\title{
Apocynin preserves glutamatergic neurons in the basolateral amygdala in mice with neonatal sevoflurane exposure
}

\author{
Zhongliang Sun ${ }^{1}$, Maiko Satomoto $^{2}$, Yushi U Adachi ${ }^{2}$, and Koshi Makita ${ }^{1}$ \\ Department of Anesthesiology, ${ }^{1}$ Graduate School of Medical and Dental Science, Tokyo Medical and Dental \\ University, Tokyo, ${ }^{2}$ Graduate School of Medicine, Nagoya University, Aichi, Japan
}

Background: Neonatal exposure to anesthetics induces neuronal apoptosis and long-term cognitive dysfunction in rodents. We showed that the nicotinamide adenine dinucleotide phosphate-oxidase inhibitor apocynin not only reduces neurotoxicity by decreasing superoxide levels and preventing mitochondrial dysfunction but also improves long-term memory impairment in neonatal mice exposed to sevoflurane. We also found that after the contextual fear conditioning test, glutamatergic neurons expressed c-Fos (neural activation) regardless of previous exposure to sevoflurane. Moreover, there were fewer c-Fos-expressing glutamatergic neurons in the basolateral amygdala (BLA) after exposure to sevoflurane than after exposure to carrier gas. In this study, we investigated whether the administration of apocynin prior to sevoflurane exposure would preserve glutamatergic neurons in the BLA.

Methods: Apocynin $(50 \mathrm{mg} / \mathrm{kg}$ ) was injected intraperitoneally into six-day-old male mice $30 \mathrm{~min}$ before $6 \mathrm{~h}$ of exposure to $3 \%$ sevoflurane or carrier gas only. The mice were allowed to mature and then were subjected to the contextual fear conditioning test. The neural activation and neuron population in the BLA were investigated $2 \mathrm{~h}$ later.

Results: Administration of apocynin prior to neonatal sevoflurane exposure not only prevented learning deficits but also preserved c-Fos-expressing glutamatergic neurons in the BLA.

Conclusions: Apocynin mitigates the cognitive impairment induced by neonatal sevoflurane exposure and preserves cFos-expressing glutamatergic neurons in the basolateral amygdala.

Key Words: Anesthesia, Brain, Neurotoxicity, Pediatrics, Sevoflurane.

Corresponding author: Maiko Satomoto, M.D., Ph.D.

Department of Anesthesiology, Graduate School of Medicine, Nagoya University, 65 Tsurumai, Showa-ku, Nagoya 466-8550, Japan

Tel: 81-52-744-2340, Fax: 81-52-744-2342

Email: satomoto@med.nagoya-u.ac.jp

ORCID: http://orcid.org/0000-0003-2768-7482

Received: December 29, 2016.

Revised: January 30, 2017 (1st); February 21, 2017 (2nd).

Accepted: February 22, 2017.

Korean J Anesthesiol 2017 June 70(3): 335-340

https://doi.org/10.4097/kjae.2017.70.3.335

\section{Introduction}

Exposure to anesthetics induces neuronal apoptosis and long-term cognitive dysfunction in neonatal rodents [1-4]. Sevoflurane is an inhaled anesthetic commonly used to induce and maintain anesthesia in pediatric patients. Recent animal studies have demonstrated that neonatal sevoflurane exposure is neurotoxic, resulting in long-term cognitive and behavioral deficits $[4,5]$.

Recently, we found that the nicotinamide adenine dinucleotide phosphate (NADPH) oxidase inhibitor apocynin reduces neurotoxicity by decreasing superoxide levels and preventing mitochondrial dysfunction [6]. Furthermore, apocynin miti-

(c) This is an open-access article distributed under the terms of the Creative Commons Attribution Non-Commercial License (http://creativecommons.org/ licenses/by-nc/4.0/), which permits unrestricted non-commercial use, distribution, and reproduction in any medium, provided the original work is properly cited. 
gates long-term memory impairment in neonatal mice exposed to sevoflurane [6]. We also found that neonatal mice exposed to sevoflurane have a reduced freezing time in the contextual fear conditioning test, probably due to decreased neuronal activation in the basolateral amygdala (BLA) [7]. A concurrent decrease in glutamatergic neurons in the BLA could explain the learning deficits caused by sevoflurane exposure [7].

Therefore, in this study, we investigated whether administration of apocynin to neonatal mice prior to sevoflurane exposure would preserve glutamatergic neurons in the BLA.

\section{Materials ans Methods}

\section{Ethical considerations and experimental mice}

Ethical approval for this study was obtained from the Animal Care and Use Committee of Tokyo Medical and Dental University (approval numbers 0140163C and 0150033A). Litters of postnatal day 5 C57BL/6 male mice (average body weight $2.7 \mathrm{~g}$ ) along with their mothers were purchased from SLC Japan, Inc., Shizuoka, Japan. The animals were housed under a 12-h light-dark cycle (lights on $08: 00$ to $20: 00)$ at $21 \pm 1^{\circ} \mathrm{C}$. Thirty of the male pups were placed under anesthesia with sevoflurane on postnatal day 6 and then returned to their original litters. The same number of pups from each litter was used for the experiments to reduce variability related to the use of different litters. At the age of 3-4 weeks, the male pups were weaned and housed four animals to a cage. The mice had ad libitum access to water and food. Behavioral testing was performed in adulthood (11-13 weeks of age). This study used as few animals as possible. Only male mice were used to avoid potential variability caused by the estrous cycle [8].

\section{Anesthesia protocol}

On postnatal day 6 , male pups (SEVO group; $\mathrm{n}=10$ ) were placed in a humid chamber $(180 \times 180 \times 200 \mathrm{~mm})$ with a mat heated to $38 \pm 1^{\circ} \mathrm{C}$, and $3 \%$ sevoflurane was delivered using a calibrated flow meter (Shimano, Tokyo, Japan) as described previously [6,7]. The gas was administered at $1 \mathrm{~L} / \mathrm{min}$ in $40 \% \mathrm{O}_{2}$ for $6 \mathrm{~h}$. Pups exposed to only $40 \% \mathrm{O}_{2}$ for $6 \mathrm{~h}$ were used as controls (NA group; $\mathrm{n}=10$ ). Thirty minutes prior to sevoflurane exposure, the NADPH oxidase inhibitor apocynin was administered intraperitoneally at $50 \mathrm{mg} / \mathrm{kg}$ for a total volume of $10 \mu \mathrm{l}$ (apocy$\operatorname{nin}+$ SEVO group; $\mathrm{n}=10$ ) as previously described [6]. The pups from each experimental group were returned to their litter and allowed to mature.

\section{Fear conditioning test}

At the age of 11-13 weeks, mice were subjected to the con- textual fear conditioning test. Their movement was monitored using a computerized video tracking system designed by O'Hara \& Co., Ltd. (Tokyo, Japan), as described previously [6,7]. The test consisted of three conditioned-unconditioned stimulus pairings separated by 1-min intervals. Each pairing was as follows: unconditioned stimulus, $0.5-\mathrm{mA}$ foot shock of 1 -s duration; and conditioned stimulus, $60-\mathrm{dB}$ white noise of 20 -s duration. The unconditioned stimulus was delivered during the last seconds of the conditioned stimulus. The contextual test was performed $24 \mathrm{~h}$ after conditioning. The duration of freezing in response to the stimuli was automatically recorded to measure fear memory. The freezing time during total stimulation ( $5 \mathrm{~min}$ ) was presented as the freezing percentage, which was compared between each group.

\section{Tissue preparation and histopathologic evaluation}

Two hours after the contextual fear conditioning test, the mice ( $n=6$ in each group) were deeply anesthetized with pentobarbital (50 mg/kg, intraperitoneal injection) and transcardially perfused with $4 \%$ paraformaldehyde (PFA) for $5 \mathrm{~min}$. Thereafter, their brain tissue was removed and postfixed in the same buffer overnight at $4^{\circ} \mathrm{C}$. Fifty-micrometer-thick floating coronal vibratome sections at $250-\mu \mathrm{m}$ intervals (starting at bregma 2.20 $\mathrm{mm}$ to bregma $-2.70 \mathrm{~mm}$ ) were obtained for immunohistochemistry as previously described [7].

c-Fos staining was performed to investigate neuronal activity after the fear conditioning test. Sections were washed with phosphate-buffered saline (PBS) with $0.3 \%$ triton three times (10 min each), and endogenous peroxidase activity was blocked with $1 \%$ $\mathrm{H}_{2} \mathrm{O}_{2}$ (30 min) [9-11]. Then, the sections were incubated with anti-c-Fos antiserum (1:1000; Santa Cruz Biotechnology, Santa Cruz, Dallas, TX, USA) diluted with 1\% blocking reagent in PBS with Tween 20 (Katayama Chemistry, Osaka, Japan) overnight at $4^{\circ} \mathrm{C}$. Next, the sections were incubated with peroxidase-conjugated secondary antibodies (1:1000; Abcam, Inc., Cambridge, $\mathrm{UK}$ ) for $2 \mathrm{~h}$ at room temperature and washed with PBS. The cFos signal was visualized using a 3,3'-Diaminobenzidine (DAB)nickel substrate (0.05\% DAB, 0.05\% $\mathrm{NiSO}_{4}, 0.015 \% \mathrm{H}_{2} \mathrm{O}_{2}, 0.05 \%$ $1 \mathrm{M}$ Tris- $\mathrm{HCl}, \mathrm{pH}$ 6.7). The sections were then mounted on slides, brushed, and dried overnight. The number of c-Fosexpressing cells in the BLA was counted as described previously [7]. Briefly, four sections in the BLA from each group were carefully matched among subjects using anatomical landmarks from a brain atlas. The images were counted by a single experimenter who was blind to the treatment conditions. The number of $\mathrm{c}$ Fos-expressing cells in the BLA was stated as the number of cFos-expressing cells $/ 10^{-1} \mathrm{~mm}^{2}$ [12].

To investigate the neuron type and distribution of cells expressing c-Fos, the sections were co-incubated with anti-c-Fos 
antiserum and monoclonal mouse anti-Glutamic Acid Decarboxylase (GAD)67 (1:200, MAB5406; Chemicon, Temecula, CA, USA) as the GABAergic neuron marker, or with polyclonal goat anti-Excitatory Amino Acid Carrier 1 (EAAC1) (1:1000, AB1520; Chemicon) as the glutamatergic neuron marker, and then diluted in $1 \%$ blocking reagent in PBS with Tween 20 overnight at $4^{\circ} \mathrm{C}$. Next, the sections were washed with PBS and co-incubated with Alexa-594-conjugated anti-rabbit IgG (1:1000; Invitrogen, Carlsbad, CA, USA) and Alexa-488 (1:1000; Invitrogen)-conjugated anti-mouse IgG or Alexa-488-conjugated anti-goat IgG (1:1000; Invitrogen) secondary antibody for $2 \mathrm{~h}$ at room temperature. Sections were counterstained with 4,6-diamidino-2-phenylindole (DAPI; Thermo Fisher Scientific Corp., Carlsbad, CA, USA) and then mounted on a slide with Fluoromount (Diagnostic BioSystems, Pleasanton, CA, USA). The positive cells in the BLA of each group were counted in a blind manner as previously described [7]. Briefly, positive cells in the BLA were counted and the four sections were fluorescently stained. The fluorescent im- ages were examined with a microscope using the Zeiss LSM Image Browser (Carl Zeiss MicroImaging, Jena, Germany). Settings were adjusted based on the fluorescence intensity of tissue from the control group and were identical for all sections.

\section{Statistical analysis}

Statistical analysis was performed using the SPSS statistical software package (SPSS ver. 24.0; IBM, Corp., Armonk, NY, USA). The Kruskal-Wallis omnibus test was used to compare data, followed by Bonferroni post tests. Values of $\mathrm{P}<0.05$ were considered statistically significant, and data are presented as mean \pm SEM.

\section{Results}

\section{Apocynin preserved c-Fos expression in the BLA}

c-Fos staining was performed to investigate neuronal activ-
A

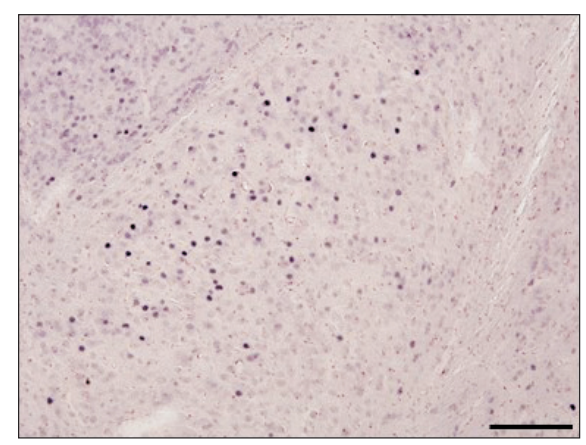

B

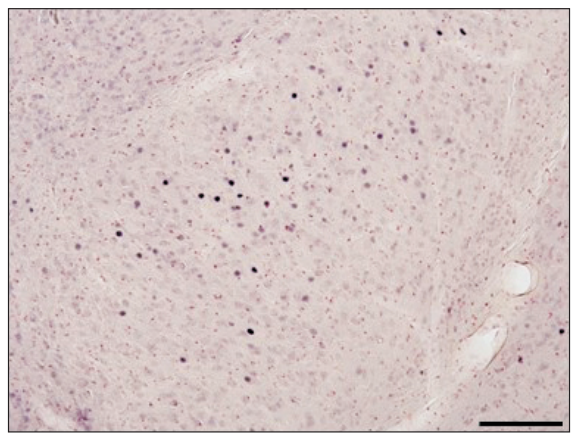

C

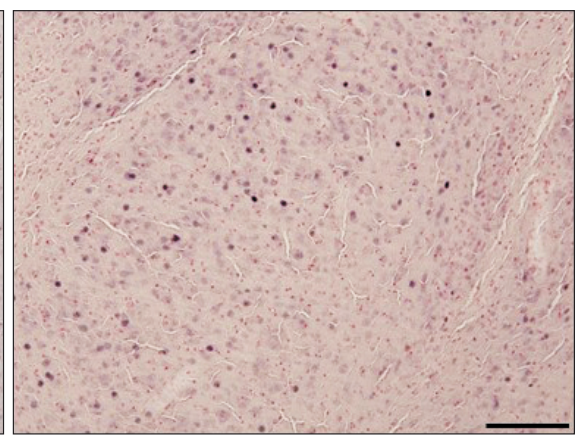

D

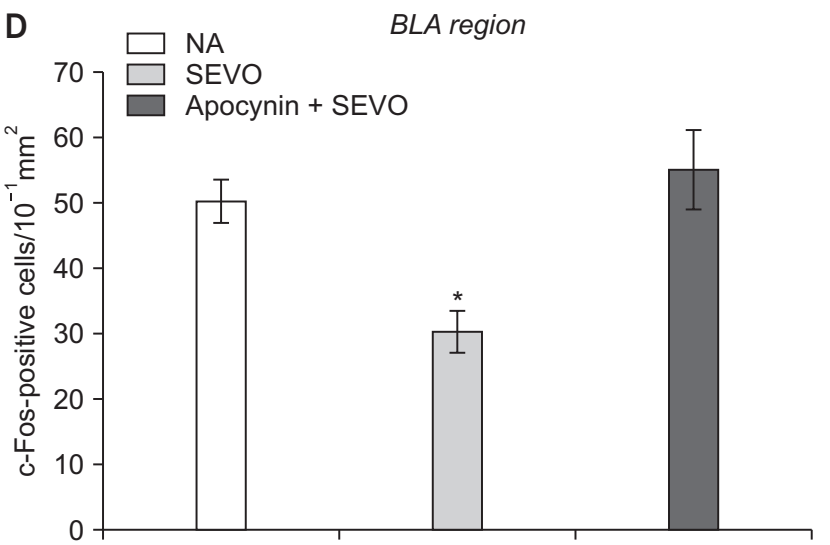

E

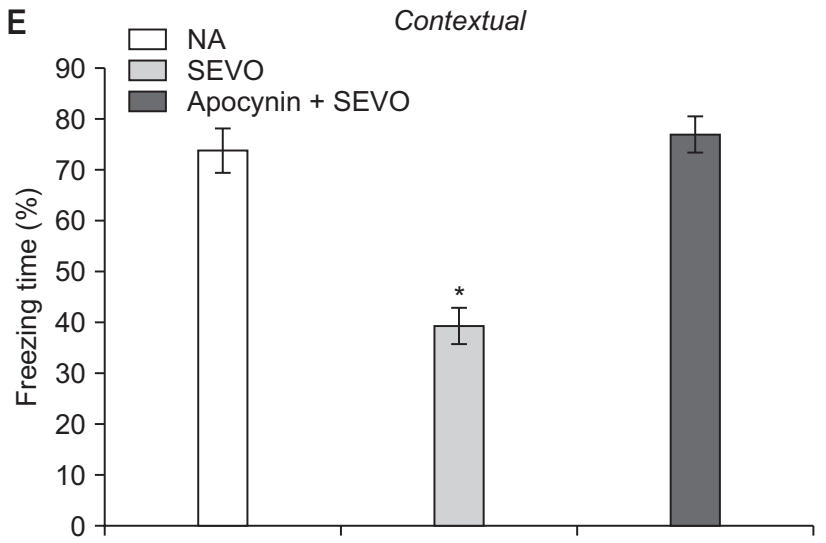

Fig. 1. The c-Fos expression in the basolateral amygdala (BLA). (A) NA group; (B) SEVO group; (C) apocynin + SEVO group. Black dots indicate c-Fos-positive cells. Scale bar: $100 \mu \mathrm{m}$. (D) The number of c-Fos-expressing cells in the BLA is expressed as the number of c-Fos-expressing cells $/ 10^{-1}$ $\mathrm{mm}^{2}$. The expression of c-Fos in the BLA was reduced in the SEVO group (NA group vs. SEVO group, $\mathrm{P}=0.046$ ), and administration of apocynin maintained c-Fos expression at a normal level (SEVO group vs. apocynin + SEVO group, $\mathrm{P}=0.046$ ); $\mathrm{n}=6$ for each group. ${ }^{*} \mathrm{P}<0.05$. (E) Neonatal sevoflurane exposure induced long-term cognitive impairment, as indicated by reduction in the freezing response in the contextual fear conditioning test in the SEVO group (NA group vs. SEVO group, $\mathrm{P}=0.001$ ). Administration of apocynin prevented neonatal sevoflurane exposure-induced learning deficits (apocynin + SEVO group vs. SEVO group, $\mathrm{P}=0.022) ; \mathrm{n}=10$ for each group. ${ }^{*} \mathrm{P}<0.05$. 
ity after the fear conditioning test. c-Fos expression in the BLA was lower in the SEVO group than in the NA group (Fig. 1) [7]. Apocynin treatment preserved neuronal activity by maintaining c-Fos expression in the BLA (Fig. 1D, SEVO vs. apocynin + SEVO, $\mathrm{P}=0.046)$.

\section{Evaluation of long-term memory}

Sevoflurane exposure significantly reduced the freezing time in the contextual fear conditioning test, while the administration of apocynin increased long-term memory (Fig. 1E, SEVO vs. Apocynin + SEVO, $\mathrm{P}=0.022$ ), as previously described [6].

\section{Apocynin preserved glutamatergic neurons in the BLA}

Recently, we showed that the cells expressing c-Fos in the
BLA were glutamatergic neurons (Fig. 2) [7]. There were fewer c-Fos-expressing glutamatergic BLA neurons in the SEVO group than in the NA group, as previously described (Fig. 1) [7]. Apocynin treatment preserved neuronal activity by maintaining the glutamatergic neurons in the BLA (Fig. 3B, SEVO vs. Apocynin + SEVO, $\mathrm{P}=0.014)$. Recently, we found that the NADPH oxidase inhibitor apocynin reduces neurotoxicity by decreasing the superoxide levels and preventing mitochondrial dysfunction [6]. Furthermore, apocynin improved long-term memory impairment in neonatal mice exposed to sevoflurane [6]. Together with the results of our recent studies, this study demonstrated that the administration of apocynin not only prevents the neuroapoptosis that occurs immediately after neonatal sevoflurane exposure, but also preserves the glutamatergic neurons in the BLA long after sevoflurane exposure. The number of GAD67-positive cells remained unchanged in both groups, indicating that the number
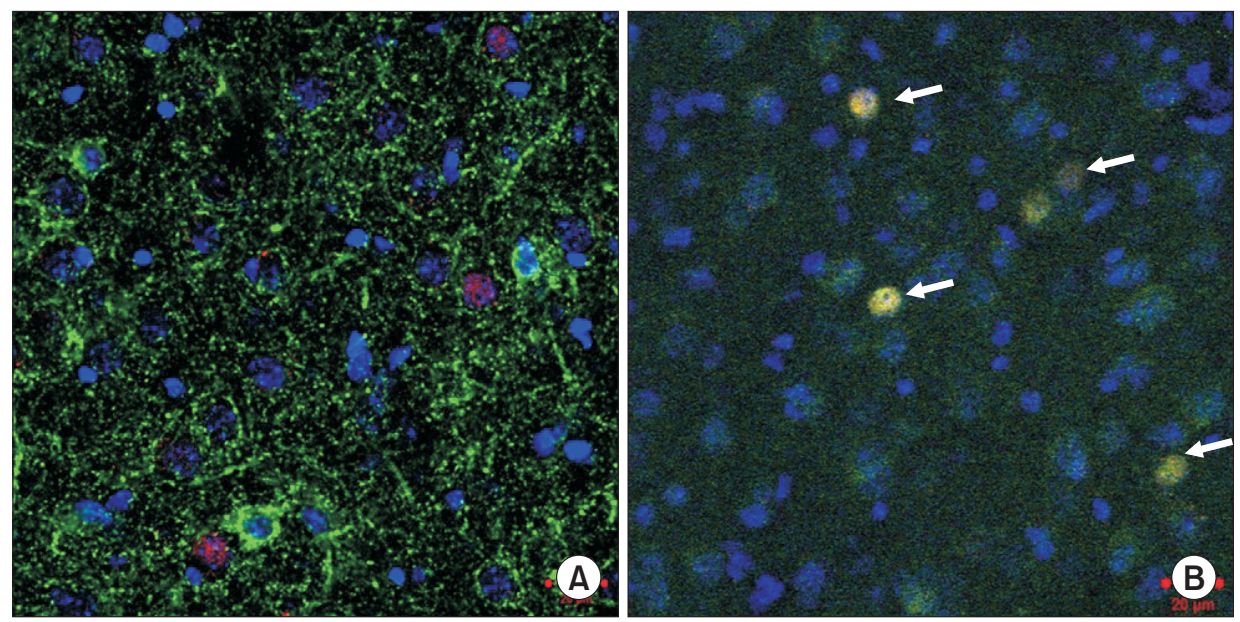

Fig. 2. Stained images: (A) GAD67 (green)/c-Fos (red) was not co-located with c-Fos. (B) EAAC1 (green)/c-Fos (red) in the BLA after the contextual fear conditioning test. EAAC1 was co-located with c-Fos (white arrow). These results indicated that the glutamatergic neurons in the BLA were activated during the fear conditioning test, but the GABAergic neurons were not. Blue dots: DAPIpositive cells. Scale bar: $20 \mu \mathrm{m}$.
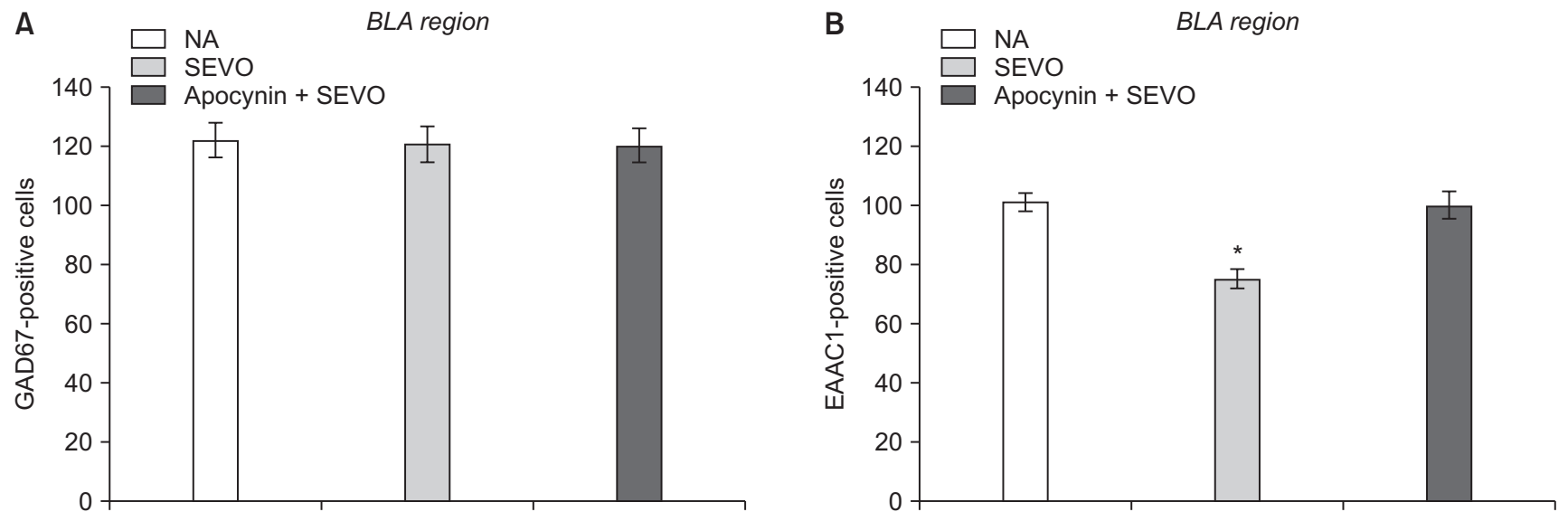

Fig. 3. The neuron distribution in the BLA. (A) The number of GAD67-positive cells; (B) the number of EAAC1-positive cells. The number of GAD67-positive cells in the groups remained unchanged, indicating that the number of GABAergic neurons was not affected by neonatal sevoflurane exposure. The number of EAAC1-positive cells was significantly decreased in the SEVO group (NA group vs. SEVO group, $\mathrm{P}=0.014$ ), indicating that the decreased density of glutamatergic neurons in the BLA is related to long-term learning deficits in mice exposed to sevoflurane as neonates. Administration of apocynin preserved glutamatergic neurons (SEVO group vs. apocynin + SEVO group, $\mathrm{P}=0.014$ ); $\mathrm{n}=6$ for each group. ${ }^{*} \mathrm{P}<0.05$. 
of GABAergic neurons was not affected by neonatal sevoflurane exposure (Fig. 3A).

\section{Discussion}

In this study, we found that apocynin preserved c-Fos expression and glutamatergic neurons in the BLA. These results, along with our previous findings, indicate that the administration of apocynin prevents long-term memory loss in neonatal mice exposed to sevoflurane by preserving glutamatergic neurons in the BLA.

Recent studies have shown that sevoflurane exposure induces widespread apoptosis in the brain of neonates, causing severe neuronal damage in the hippocampus and amygdala, which are associated with learning and memory [5,9]. Specifically, after general anesthesia, glutamatergic and GABAergic neurons undergo anesthesia-induced apoptosis [10]. GABAergic neurons undergo a switch from excitatory to inhibitory roles during development [11]. In a previous study, we showed that sevoflurane exposure did not affect the number of GABAergic neurons in the BLA in adult mice and that reduced glutamatergic neurons in the BLA led to learning deficits [7].

We found that NADPH oxidase activation plays an important role in sevoflurane-induced neurotoxicity and long-term cognitive impairment [6]. Neonatal sevoflurane exposure increased NADPH oxidase subunit expression, resulting in superoxide overproduction, mitochondrial dysfunction, and apoptosis. Administration of the NADPH oxidase inhibitor apocynin prior to sevoflurane exposure prevents long-term cognitive impairment seen in adulthood. Recent animal studies have identified several therapeutic strategies that prevent the long-term learning deficits induced by neonatal sevoflurane exposure [6,13-15]. However, these studies mainly focused on anti-apoptotic effects, rather than the prevention of anesthesia-induced long-term learning deficits [6,13-15].

Recently, we found that a difference in c-Fos expression in the BLA after the fear conditioning test was associated with learning deficits induced by neonatal sevoflurane exposure and that c-Fos-expressing glutamatergic neurons within the amygdala were decreased in neonatal mice exposed to sevoflurane [7]. Expression of c-Fos reflects the neural activity after behavioral tests [16-18]. Learning involves many regions of the brain [16,19-21]. The BLA plays a role in the acquisition and expression of learned fear $[22,23]$. We found that a reduction in BLA glutamatergic neurons was associated with a decreased freezing time during the contextual fear conditioning test in mice exposed to sevoflurane as neonates, and that the administration of apocynin prior to sevoflurane exposure preserved the glutamatergic neurons in the BLA. We did not investigate which types of neuron tend to undergo sevoflurane-induced cell death. It may be that glutamatergic neurons are susceptible to neonatal sevoflurane exposure, and apocynin-rescued glutamatergic neurons may reduce excessive superoxide concentrations.

As our recent studies indicated, 3\% sevoflurane exposure does not cause adverse cardiovascular or respiratory effects in neonatal pups [5,7]. The current study did not include mice administered only apocynin because apocynin alone does not induce neuroapoptosis or learning deficits in the fear conditioning test [6]. Following up on our previous studies, the current study investigated only neuronal activation in the BLA.

In conclusion, this study showed that the administration of apocynin preserved glutamatergic neurons in the basolateral amygdala of neonatal mice exposed to sevoflurane. We reported previously that apocynin prevents the decreased freezing time during the fear-conditioning test in mice exposed to sevoflurane as neonates. Therefore, apocynin may prevent neuronal apoptosis and subsequent cognitive impairment by preserving the glutamatergic neurons in the basolateral amygdala.

\section{Acknowledgments}

We thank Ayumi Sakata (Diversity Diamond Unit, Tokyo Medical and Dental University, Tokyo, Japan) for her excellent technical help with this study.

\section{References}

1. Jevtovic-Todorovic V, Hartman RE, Izumi Y, Benshoff ND, Dikranian K, Zorumski CF, et al. Early exposure to common anesthetic agents causes widespread neurodegeneration in the developing rat brain and persistent learning deficits. J Neurosci 2003; 23: 876-82.

2. Young C, Jevtovic-Todorovic V, Qin YQ, Tenkova T, Wang H, Labruyere J, et al. Potential of ketamine and midazolam, individually or in combination, to induce apoptotic neurodegeneration in the infant mouse brain. Br J Pharmacol 2005; 146: 189-97.

3. Brambrink AM, Evers AS, Avidan MS, Farber NB, Smith DJ, Zhang X, et al. Isoflurane-induced neuroapoptosis in the neonatal rhesus macaque brain. Anesthesiology 2010; 112: 834-41.

4. Zheng H, Dong Y, Xu Z, Crosby G, Culley DJ, Zhang Y, et al. Sevoflurane anesthesia in pregnant mice induces neurotoxicity in fetal and offspring mice. Anesthesiology 2013; 118: 516-26.

5. Satomoto M, Satoh Y, Terui K, Miyao H, Takishima K, Ito M, et al. Neonatal exposure to sevoflurane induces abnormal social behaviors and deficits in fear conditioning in mice. Anesthesiology 2009; 110: 628-37. 
6. Sun Z, Satomoto M, Adachi YU, Kinoshita H, Makita K. Inhibiting NADPH oxidase protects against long-term memory impairment induced by neonatal sevoflurane exposure in mice. Br J Anaesth 2016; 117: 80-6.

7. Satomoto M, Sun Z, Adachi YU, Makita K. Neonatal sevoflurane exposure induces adulthood fear-induced learning disability and decreases glutamatergic neurons in the basolateral amygdala. J Neurosurg Anesthesiol 2016 [Epub ahead of print].

8. Milad MR, Igoe SA, Lebron-Milad K, Novales JE. Estrous cycle phase and gonadal hormones influence conditioned fear extinction. Neuroscience 2009; 164: 887-95.

9. Zelikowsky M, Hersman S, Chawla MK, Barnes CA, Fanselow MS. Neuronal ensembles in amygdala, hippocampus, and prefrontal cortex track differential components of contextual fear. J Neurosci 2014; 34: 8462-6.

10. Zhou ZW, Shu Y, Li M, Guo X, Pac-Soo C, Maze M, et al. The glutaminergic, GABAergic, dopaminergic but not cholinergic neurons are susceptible to anaesthesia-induced cell death in the rat developing brain. Neuroscience 2011; 174: 64-70.

11. Rivera C, Voipio J, Payne JA, Ruusuvuori E, Lahtinen H, Lamsa K, et al. The K+/Cl- co-transporter KCC2 renders GABA hyperpolarizing during neuronal maturation. Nature 1999; 397: 251-5.

12. Milanovic S, Radulovic J, Laban O, Stiedl O, Henn F, Spiess J. Production of the Fos protein after contextual fear conditioning of C57BL/6N mice. Brain Res 1998; 784: 37-47.

13. Cao W, Pavlinec C, Gravenstein N, Seubert CN, Martynyuk AE. Roles of aldosterone and oxytocin in abnormalities caused by sevoflurane anesthesia in neonatal rats. Anesthesiology 2012; 117: 791-800

14. Shen X, Dong Y, Xu Z, Wang H, Miao C, Soriano SG, et al. Selective anesthesia-induced neuroinflammation in developing mouse brain and cognitive impairment. Anesthesiology 2013; 118: 502-15.

15. Yonamine R, Satoh Y, Kodama M, Araki Y, Kazama T. Coadministration of hydrogen gas as part of the carrier gas mixture suppresses neuronal apoptosis and subsequent behavioral deficits caused by neonatal exposure to sevoflurane in mice. Anesthesiology 2013; 118: 10513 .

16. Marota JJ, Crosby G, Uhl GR. Selective effects of pentobarbital and halothane on c-fos and jun-B gene expression in rat brain. Anesthesiology 1992; 77: 365-71.

17. Lanuza E, Moncho-Bogani J, Ledoux JE. Unconditioned stimulus pathways to the amygdala: effects of lesions of the posterior intralaminar thalamus on foot-shock-induced c-Fos expression in the subdivisions of the lateral amygdala. Neuroscience 2008; 155: 959-68.

18. Lelos MJ, Good MA. c-Fos expression reveals aberrant neural network activity during cued fear conditioning in APPswe transgenic mice. Neurobiol Learn Mem 2012; 98: 1-11.

19. Costanzi M, Saraulli D, Cannas S, D'Alessandro F, Florenzano F, Rossi-Arnaud C, et al. Fear but not fright: re-evaluating traumatic experience attenuates anxiety-like behaviors after fear conditioning. Front Behav Neurosci 2014; 8: 279.

20. Marek R, Strobel C, Bredy TW, Sah P. The amygdala and medial prefrontal cortex: partners in the fear circuit. J Physiol 2013; 591: 2381-91.

21. Lelos MJ, Good MA. $\beta$-Amyloid pathology alters neural network activation during retrieval of contextual fear memories in a mouse model of Alzheimer's disease. Eur J Neurosci 2014; 39: 1690-703.

22. Sierra-Mercado D, Padilla-Coreano N, Quirk GJ. Dissociable roles of prelimbic and infralimbic cortices, ventral hippocampus, and basolateral amygdala in the expression and extinction of conditioned fear. Neuropsychopharmacology 2011; 36: 529-38.

23. Vazdarjanova A, Cahill L, McGaugh JL. Disrupting basolateral amygdala function impairs unconditioned freezing and avoidance in rats. Eur J Neurosci 2001; 14: 709-18. 